\title{
A method for matching the eigenfrequencies of longitudinal and torsional vibrations in a hybrid piezoelectric motor
}

\author{
Youping $\mathrm{Yi}^{\mathrm{a}, *}$, Wolfgang Seemann ${ }^{\mathrm{b}}$, Rainer Gausmann ${ }^{\mathrm{c}}$, Jue Zhong ${ }^{\mathrm{a}}$ \\ ${ }^{a}$ College of Electrical and Mechanical Engineering, Central South University, 410083 Changsha, China \\ ${ }^{\mathrm{b}}$ Institut für Technische Mechanik, Universität Karlsruhe (TH), 76128 Karlsruhe, Germany \\ ${ }^{\mathrm{c}}$ AG Maschinendynamik, Universität Kaiserslautern, 67653 Kaiserslautern, Germany
}

Received 14 April 2004; received in revised form 21 September 2004; accepted 27 January 2006

Available online 3 April 2006

\begin{abstract}
A hybrid piezoelectric motor is investigated, which uses a longitudinal transducer and a tangential transducer to generate the desired elliptical trajectories on the surface of the stator. In general, such a motor consists of one stator and one rotor. The frequency at which it is driven is the resonance frequency of the tangential motion of the stator. For the purpose of enlarging the micro displacement generated by the ultrasonic vibration and obtaining a large torque and a higher efficiency, the resonance frequency of the longitudinal vibration and torsional vibration should coincide. However, it is difficult to match these two resonance frequencies of the two transducers with an identical geometry size. In this paper, a new prototype of a hybrid piezoelectric motor is proposed, in which two stators are used to increase the contact area between the rotor and the stator. Besides, two adjusting rings are added to the stators to adjust the resonance frequencies of both vibrations in such a way that they coincide. A theoretical model and a FE model are used to optimize the geometry of the motor and to investigate the effects of matching the eigenfrequencies.
\end{abstract}

(C) 2006 Published by Elsevier Ltd.

\section{Introduction}

Since the hybrid piezoelectric motor has been first proposed in the 1980s, it attracts considerable attention due to the properties, which are much better than the properties of traveling wave motors: it has a larger output torque and a higher efficiency [1-3]. Unlike the traveling wave ultrasonic motor [4], this motor uses two types of PZT transducers, one is the longitudinal transducer, which generates the longitudinal motion; the other is the tangential transducer, which excites the torsional motion. For the case of a temporal phase shift between the vibrations of these two transducers of $90^{\circ}$, the surface points of the stator move on elliptic trajectories [5].

In the past these hybrid piezoelectric motors were excited with a frequency, which corresponds to the eigenfrequency of the torsional vibration mode. In order to obtain a larger output torque and a higher

\footnotetext{
*Corresponding author. Fax: + 8607318710201.

E-mail addresses: yyp@mail.csu.edu.cn (Y. Yi), Seemann@itm.uni-karlsruhe.de (W. Seemann), Rainer.Gausmann@gmx.net (R. Gausmann), jzhong@mail.csu.edu.cn (J. Zhong).
} 
efficiency, not only the torsional mode should be driven in resonance but both transducers. As the transducers have to be excited with the same frequency they should have the same eigenfrequency. However, it is difficult to match these two resonance frequencies of the two transducers. This can be seen easily if the stator is modeled as a rod [6]. In such a rod the speeds for torsional and longitudinal waves are different and therefore, the resonance frequencies are different in general.

Previous papers have proposed some methods to match the eigenfrequencies of the longitudinal transducer and the torsional transducer such as adding a mass to the end of the stator or changing the area of cross section of the stator, etc [7-10]. These measures can change the eigenfrequencies of these two types of vibration and obtain good results. But there are still problems when these methods are used in practice. Not only the stator should be fabricated many times, but also the eigenfrequency could not be adjusted any more when the motor has been assembled. Therefore, a possibility to match the eigenfrequencies of these two types of vibration is the key to increase the output torque and the efficiency of the hybrid piezoelectric motor.

Like for a typical hybrid piezoelectric motor, one stator and one rotor are used in the model. To obtain a large torque, the longitudinal and tangential PZT should be placed on the node of the corresponding vibration mode. As vibration modes the first longitudinal mode and the first torsional mode are used. In a very rough model, the stator may be approximated by an elastic cylindrical rod. In the case of same boundary conditions, the first eigenfrequency of the torsional vibration is always lower than that of the longitudinal vibration, because the speed of torsional waves is slower than the speed of longitudinal waves. If the geometry of the motor is changed, for example the length of the cylinder, both the eigenfrequency of the torsional vibration and the eigenfrequency of the longitudinal vibration are also changed. As the speed of waves does not depend on the diameter for a circular cross section, the ratio of the eigenfrequencies does not change with length. However, an increasing length leads to a decrease in the first eigenfrequency.

In this paper, a new prototype of hybrid piezoelectric motor is proposed, in which two stators are used to increase the contact area between the rotor and the stator, see Fig. 1. To match the eigenfrequencies of two vibrations, two adjusting rings are added to the outside of two stators.

The working principle of the present motor can be illustrated by Fig. 2. The temporal phase shift is assumed $\pi / 2$. When the voltage $V_{\mathrm{T}}$ applied to T-PZT reaches its peak value, the torsional displacement obtains the maximum, while the longitudinal transducer has no deformation. Two stators are in loose contact with the rotor (see Fig. 2a). As the voltage $V_{\mathrm{L}}$ applied to L-PZT increases and reaches the peak, the longitudinal transducer extends and two stators are in firm contact with the rotor (see Fig. 2b). At this moment, the tangential velocity of points at the interface between rotor and stator reaches the maximum, and the rotor will rotate undergoing the friction forces. In Fig. 2c, the torsional deformation of the stators will be along the same direction, but the longitudinal transducer will contract and its deformation decreases to zero. Two stators are in loose contact with the rotor again. Finally, the longitudinal transducer will continue to contract while the torsional transducer begins to deform in reverse direction, and the rotor will separate from two stators. Due to the inertia, the rotor will rotate in the same direction(see Fig. 2d). In such a cycle, the rotor has rotated by a small angle. With the high frequency AC voltages excitation, a continuous rotary motion occurs in this motor.

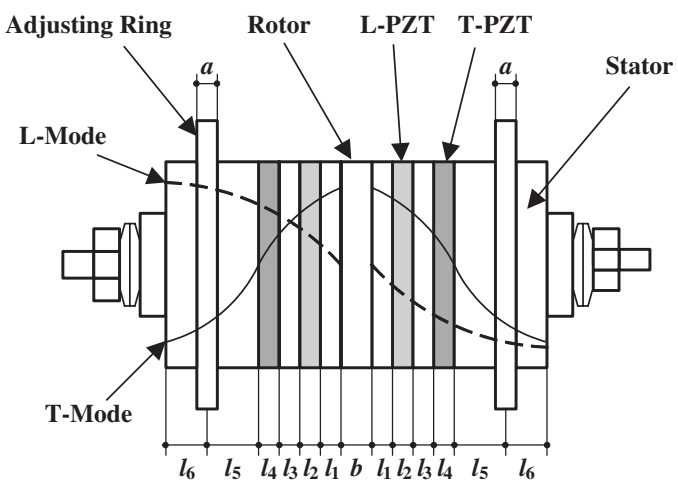

Fig. 1. Basic parts of the motor and vibration modes used to run the motor. 

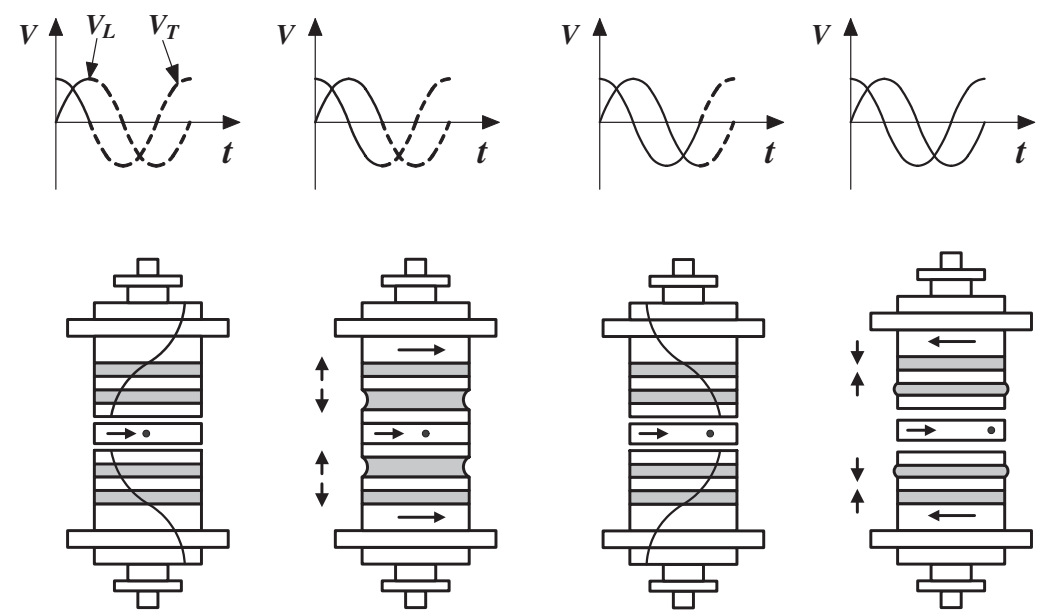

(a)

(b)

(c)

(d)

Fig. 2. Working principle of the present piezoelectric motor.

Compared with existing motors, this motor has a different structure and its own properties. The rotor is placed in the middle of the motor and larger dynamical normal forces may be generated by longitudinal vibration of opposite stators. In addition, the contact area between the rotor and the stator is two times as that of the previous motors. This is useful to enhance the output torque of the motor. Due to the symmetric structure, the boundary condition of longitudinal vibration and torsional vibration are different from that of the previous motors. For the longitudinal vibration in a stator, it can be assumed that one end is free and the other is fixed. In comparison with the longitudinal vibration, both end of the stator are free for the torsional vibration. For the operation of the motor the first mode for torsional vibration and the first mode for longitudinal vibration are used. It should be noticed that the phase shift of vibrations between two stators is $180^{\circ}$. Besides, an adjusting ring is used in this motor. The adjusting ring fixed to each stator has two functions. On one hand, it can support the motor and it can easily be fixed. It is worth mentioning that the supporting position of the motor has some influence on the eigenfrequency of the motor. So this structure is helpful for practical applications. On the other hand, the ring can be used to adjust the eigenfrequencies of longitudinal and torsional vibration via changing the mass or position of the ring to match these two eigenfrequencies.

Based on this prototype motor, a theoretical model is proposed to investigate the characteristics of the motor and the operatability of the adjusting rings. A prototype motor is designed and fabricated according to the analytical results.

\section{Theoretical model}

In order to analyze the eigenfrequencies of the stator and the function of the adjusting ring, the necessary theoretical background of the electromechanical behavior is given first. Therefore, a theoretical model is proposed in form of a cylindrical bar with an additional rigid ring. The equations of motion for longitudinal and torsional vibration are derived. Due to the fact that in a linear analysis longitudinal vibration and torsional vibration are uncoupled, two analytical models one for the longitudinal mode and one for the torsional mode are derived. It should be mentioned that in this paper a ring-shaped PZT is used as longitudinal transducer and the shear element PZT is bonded with epoxy adhesive as torsional transducer. The physical models of these two vibrations have the same form, but the coordinate systems and the boundary conditions are different. The direction of electric field for the L-mode PZT is the same as that of the polarization, while the direction of electric field for the T-mode PZT is normal to that of the polarization, see Figs. 3 and 4 . 


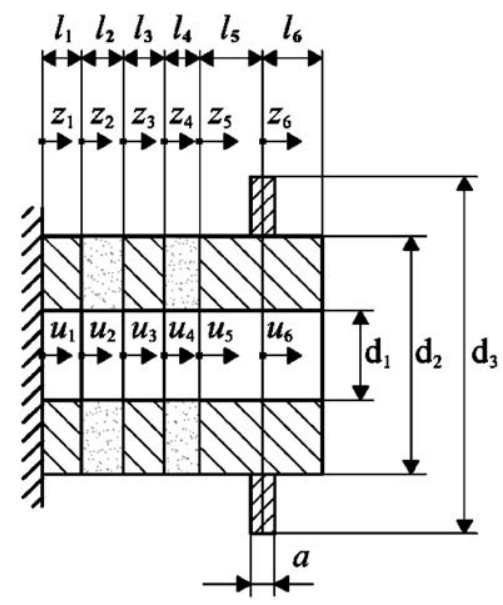

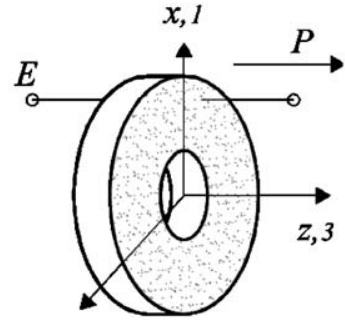

$y, 2$

Fig. 3. Physical model for longitudinal vibration.
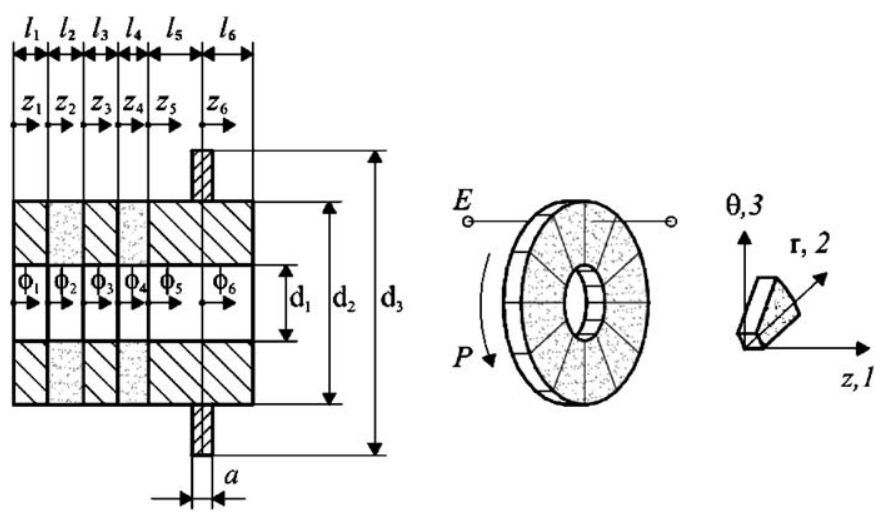

Fig. 4. Physical model for torsional vibration.

\subsection{Constitutive equations}

In the following the constitutive equations for the material used in the rod are given. As these equations are well known for a pure elastic material (brass), only the constitutive equations are given for the piezoceramic material, which excites both the longitudinal and the torsional vibrations. For each type of excitation, a corresponding material with a special poling direction is necessary. The piezoceramic exciting the longitudinal vibration has a poling direction in direction of the axis of the rod. The electric field generated by an electric voltage between two electrodes is also in direction of the rod. In this case the constitutive equations read

$$
\begin{gathered}
{\left[\begin{array}{l}
T_{1} \\
T_{2} \\
T_{3}
\end{array}\right]=} \\
{\left[\begin{array}{lll}
c_{11}^{E} & c_{12}^{E} & c_{13}^{E} \\
c_{12}^{E} & c_{11}^{E} & c_{13}^{E} \\
c_{13}^{E} & c_{13}^{E} & c_{33}^{E}
\end{array}\right]\left[\begin{array}{l}
S_{1} \\
S_{2} \\
S_{3}
\end{array}\right]-\left[\begin{array}{ccc}
0 & 0 & e_{31} \\
0 & 0 & e_{31} \\
0 & 0 & e_{33}
\end{array}\right]\left[\begin{array}{c}
E_{1} \\
E_{2} \\
E_{3}
\end{array}\right],} \\
D_{3}=e_{31} S_{1}+e_{31} S_{2}+e_{33} S_{3}+\varepsilon_{33}^{S} E_{3} .
\end{gathered}
$$

In these equations the symbol $c_{i j}^{E}$ corresponds to the coefficients of the stiffness matrix for constant electric field, $e_{i j}$ to the elements of the piezoelectric matrix and $\varepsilon_{33}^{S}$ to the permittivity for constant strain. The normal stresses are $T_{1}$ to $T_{3}$, the corresponding strains $S_{1}$ to $S_{3} . D_{3}$ and $E_{3}$ denote the electric displacement and the electric field in axial direction, respectively. 
Assuming that in transverse direction there are no normal stresses,

$$
T_{1}=T_{2}=0,
$$

the following relations for $T_{3}$ and $D_{3}$ can be obtained:

$$
\begin{aligned}
& T_{3}=\left(c_{33}^{E}-\frac{2\left(c_{13}^{E}\right)^{2}}{c_{11}^{E}+c_{12}^{E}}\right) S_{3}-\left(e_{33}-\frac{2 c_{13}^{E} e_{31}}{c_{11}^{E}+c_{12}^{E}}\right) E_{3}, \\
& D_{3}=\left(e_{33}-\frac{2 c_{13}^{E} e_{31}}{c_{11}^{E}+c_{12}^{E}}\right) S_{3}+\left(\varepsilon_{33}^{S}+\frac{2 e_{31}^{2}}{c_{11}^{E}+c_{12}^{E}}\right) E_{3} .
\end{aligned}
$$

Simplifying Eqs. (4) and (5) by introducing new constants, the constitutive equations for the PZT exciting the longitudinal mode reads

$$
\begin{aligned}
& T_{3}=\bar{c}_{33} S_{3}-\bar{e}_{33} E_{3}, \\
& D_{3}=\bar{e}_{33} S_{3}+\bar{\varepsilon}_{33} E_{3} .
\end{aligned}
$$

When the torsional vibration is analyzed, the stiffness constant $c_{44}^{E}$ of the longitudinal PZT should be considered. The equation of corresponding stress in tangential direction reads

$$
T_{4}=c_{44}^{E} S_{4}=G_{2} S_{4},
$$

where $G_{2}$ is the shear module of longitudinal PZT in tangential direction.

For the PZT used to excite the torsional vibration, the poling direction is in tangential direction whereas the electric field is also in axial direction of the rod. The constitutive equations for this case can be written as

$$
\begin{aligned}
& T_{5}=c_{55}^{E} S_{5}-e_{15} E_{1}, \\
& D_{1}=e_{15} S_{5}+\varepsilon_{11}^{S} E_{1} .
\end{aligned}
$$

The symbol $c_{55}^{E}, e_{15}$ and $\varepsilon_{11}^{S}$ also refer to stiffness-, piezoelectric- and permittivity constants like for the longitudinal PZT. The shear stress in tangential direction is $T_{5}$, the corresponding strain $S_{5} . D_{1}$ and $E_{1}$ denote the electric displacement and the electric field in axial direction.

When the longitudinal vibration is analyzed, the stress of the torsional PZT in axial direction should also be considered and may be written as

$$
T_{1}=c_{11}^{E} S_{1}+c_{12}^{E} S_{2}+c_{13}^{E} S_{3}
$$

Taking into account the assumption $T_{2}=T_{3}=0$ for the torsional PZT yields

$$
\begin{gathered}
S_{2}=\frac{c_{12}^{E} c_{33}^{E}-c_{13}^{E^{2}}}{c_{13}^{E^{2}}-c_{11}^{E} c_{33}^{E}} S_{1}, \\
S_{3}=\frac{c_{11}^{E} c_{13}^{E}-c_{12}^{E} c_{13}^{E}}{c_{13}^{E^{2}}-c_{11}^{E} c_{33}^{E}} S_{1} .
\end{gathered}
$$

Substituting Eqs. (12) and (13) into Eq. (11) yields

$$
T_{1}=\frac{c_{12}^{E^{2}} c_{13}^{E}-c_{11}^{E^{2}} c_{33}^{E}+2 c_{11}^{E} c_{13}^{E^{2}}-2 c_{12}^{E} c_{13}^{E^{2}}}{c_{13}^{E^{2}}-c_{11}^{E} c_{33}^{E}} S_{1}=Y_{4} S_{1},
$$

where $Y_{4}$ is the Young's modulus of the torsional PZT in axial direction. 


\subsection{Modeling of the longitudinal vibration}

The mathematical model for longitudinal vibration is based on the equation of motion for a onedimensional continuum. The balance of forces for a differential element yields

$$
T_{3}^{\prime}(z, t)-\rho \ddot{u}(z, t)=0 .
$$

In this equation ( $)^{\prime}$ corresponds to a derivative with respect to the spatial coordinate and ( $)$ to a derivative with respect to time. To derive the equation of motion for longitudinal vibration in the PZT region, the piezoelectric Eqs. (6) and (7) are used. Besides, the vanishing divergence of the electric displacement, which in the case of the present model reads

$$
\frac{\mathrm{d} D_{3}}{\mathrm{~d} z}=0
$$

is used to get an equation for the electric field $E_{3}$. Substituting Eq. (16) into Eq. (7), the result for the electric field is

$$
E_{3}^{\prime}=-\frac{\bar{e}_{33}}{\bar{\varepsilon}_{33}} \cdot S_{3}^{\prime},
$$

where the strain $S_{3}$ in longitudinal direction is given by

$$
S_{3}=u^{\prime}
$$

Substituting Eqs. (6), (17) and (18) into Eq. (15) yields

$$
\rho_{i} \ddot{u}_{i}-\left(\bar{c}_{33}+\frac{\bar{e}_{33}^{2}}{\bar{\varepsilon}_{33}}\right) u_{i}^{\prime \prime}=0, \quad i=2 .
$$

Similarly, in the pure elastic material and in the torsional PZT regions, the partial differential equation may be written as

$$
\rho_{i} \ddot{u}_{i}-Y_{i} u_{i}^{\prime \prime}=0, \quad i=1,3,4,5,6,
$$

with Young's modulus $Y_{i}$. For a time harmonic excitation with given circular frequency $\Omega$, the stationary solution is given by a time harmonic function

$$
u_{i}\left(z_{i}, t\right)=U_{i}\left(z_{i}\right) \mathrm{e}^{\mathrm{j} \Omega t},
$$

where $U_{i}\left(z_{i}\right)$ represents a function of $z$ that defines the shape of the vibration mode. Substitution of Eq. (21) into Eqs. (19) and (20) results in

$$
\begin{gathered}
U_{i}^{\prime \prime}+\left(\frac{\rho_{i} \Omega^{2}}{\bar{c}_{33}+\left(\bar{e}_{33}^{2} / \bar{\varepsilon}_{33}\right)}\right) \cdot U_{i}=0, \quad i=2, \\
U_{i}^{\prime \prime}+\left(\frac{\rho_{i} \Omega^{2}}{Y_{i}}\right) \cdot U_{i}=0, \quad i=1,3,4,5,6 .
\end{gathered}
$$

Furthermore, Eqs. (22) and (23) can be simplified as

$$
U_{i}^{\prime \prime}+\kappa_{i}^{2} U_{i}=0
$$

with

$$
\begin{gathered}
\kappa_{i}^{2}=\frac{\rho_{i} \Omega^{2}}{Y_{i}}, \quad i=1,3,4,5,6, \\
\kappa_{i}^{2}=\frac{\rho_{i} \Omega^{2}}{\bar{c}_{33}+\left(\bar{e}_{33}^{2} / \bar{\varepsilon}_{33}\right)}, \quad i=2 .
\end{gathered}
$$


For Eq. (24) the solution reads

$$
U_{i}(z)=C_{1 i} \sin \kappa_{i} z_{i}+C_{2 i} \cos \kappa_{i} z_{i}, \quad i=1, \ldots, 6,
$$

where $C_{1 i}$ and $C_{2 i}$ are integration constants which are determined by satisfying the boundary and transition conditions at the ends of the stator and between the different regions shown in Fig. 3.

In the following it is assumed that the two electrodes of the PZT in Section 2 are connected (short-circuited) and one end of stator is fixed and the other is free. Between the different sections the displacements and the stresses are continuous except at the transition where the ring is fixed. The width of the ring is neglected and the mass of the ring is considered to be a concentrated mass. Thus, the boundary and transition conditions for the problem may be written as

$$
\begin{aligned}
& U_{1}(0)=0, \quad U_{6}^{\prime}\left(l_{6}\right)=0, \quad U_{i}\left(l_{i}\right)=U_{i+1}(0) \quad(i=1, \ldots, 5), \\
& Y_{3} U_{3}^{\prime}\left(l_{3}\right)-Y_{4} U_{4}^{\prime}(0)=0, \quad Y_{4} U_{4}^{\prime}\left(l_{4}\right)-Y_{5} U_{5}^{\prime}\left(l_{5}\right)=0, \\
& Y_{1} U_{1}^{\prime}\left(l_{1}\right)-\left(\bar{c}_{33}+\frac{\bar{e}_{33}^{2}}{\bar{\varepsilon}_{33}}\right) U_{2}^{\prime}(0)+\frac{\bar{e}_{33}^{2}}{l_{2} \bar{\varepsilon}_{33}}\left[U_{2}\left(l_{2}\right)-U_{2}(0)\right]=0, \\
& Y_{3} U_{3}^{\prime}(0)-\left(\bar{c}_{33}+\frac{\bar{e}_{33}^{2}}{\bar{\varepsilon}_{33}}\right) U_{2}^{\prime}\left(l_{2}\right)+\frac{\bar{e}_{33}^{2}}{l_{5} \bar{\varepsilon}_{33}}\left[U_{2}\left(l_{2}\right)-U_{2}(0)\right]=0, \\
& Y_{5} A U_{5}^{\prime}\left(l_{5}\right)-Y_{6} A U_{6}^{\prime}(0)=m \Omega^{2} U_{6}(0),
\end{aligned}
$$

where $A$ is the area of the cross section of the stator and $m$ is the mass of the adjusting ring.

Substitution of Eq. (27) into Eq. (28) leads to a system of linear equations for the integration constants

$$
\mathbf{B C}=\mathbf{r},
$$

where $\mathbf{C}=\left(C_{11}, C_{12}, \ldots, C_{16}, C_{21}, \ldots, C_{26}\right)^{\mathrm{T}}$ and $\mathbf{B}$ is the coefficient matrix. In order to be able to solve Eq. (27), the condition

$$
\operatorname{det}(\mathbf{B})=0
$$

should be satisfied, because Eq. (30) corresponds to the characteristic equation of the eigenvalue-problem with which the eigenfrequencies for longitudinal vibration can be determined. This can be done for example with MAPLE or with any other program. If the excitation frequency coincides with an eigenfrequency, the undamped system will vibrate with infinitely large amplitudes.

\subsection{Modeling of torsional vibration}

The mathematical model for the torsional vibration can be obtained in a similar way. The torque of an arbitrary cross section in the stator can be written as

$$
M=\int_{A} \operatorname{Tr} \mathrm{d} A .
$$

The balance equation for the torque yields

$$
M^{\prime}=\rho I_{p} \ddot{\phi}
$$

in which $I_{p}=\int_{A} r^{2} \mathrm{~d} A$ represents the polar moment of inertia of the stator. The relation between the angular displacement $\phi$ and the strain in tangential direction is given by

$$
S=r \phi^{\prime} .
$$

Substitution of Eq. (33) and of the constitutive equation into Eq. (32) leads to the partial differential equation for the pure elastic material and for the longitudinal PZT regions

$$
\rho_{i} \ddot{\phi}_{i}-G_{i} \phi_{i}^{\prime \prime}=0, \quad i=1,2,3,5,6 .
$$


For the torsional PZT region, from

$$
\frac{\mathrm{d} D_{1}}{\mathrm{~d} z}=0
$$

it follows

$$
D_{1}^{\prime}=c_{15}^{E} S_{5}^{\prime}+\varepsilon_{11}^{S} E_{1}^{\prime}=0
$$

and therefore

$$
E_{1}^{\prime}=-\frac{e_{15}}{\varepsilon_{11}^{S}} S_{5}^{\prime}
$$

so that

$$
T_{5}^{\prime}=c_{55}^{E} S_{5}^{\prime}-e_{15} E_{1}^{\prime}
$$

leads to

$$
T_{5}^{\prime}=\left(c_{55}^{E}+\frac{e_{15}^{2}}{\varepsilon_{11}^{S}}\right) S_{5}^{\prime}=\left(c_{55}^{E}+\frac{e_{15}^{2}}{\varepsilon_{11}^{S}}\right) r \phi^{\prime \prime} .
$$

Considering Eqs. (32) and (39), the partial differential equation for the torsional PZT is obtained

$$
\rho_{i} \ddot{\phi}_{i}-\left(c_{55}^{E}+\frac{e_{15}^{2}}{\varepsilon_{11}^{S}}\right) \phi_{i}^{\prime \prime}=0, \quad i=4 .
$$

Like for longitudinal vibration the stationary solution is also given by a time harmonic function

$$
\phi_{i}\left(z_{i}, t\right)=\Phi_{i}\left(z_{i}\right) \mathrm{e}^{\mathrm{j} \Omega t},
$$

where $\Phi_{i}\left(z_{i}\right)$ represents a function of $z$ that defines the shape of the natural mode of vibration. The symbol $\Omega$ is the circular frequency. Substitution of Eq. (41) into Eqs. (34) and (40) results in

$$
\begin{gathered}
\Phi_{t}^{\prime \prime}+\left(\frac{\rho_{i} \Omega^{2}}{G_{i}}\right) \cdot \Phi_{i}=0, \quad i=1,2,3,5,6, \\
\Phi_{i}^{\prime \prime}+\left(\frac{\rho_{i} \Omega^{2}}{c_{55}^{E}+\left(e_{15}^{2} / \varepsilon_{11}^{S}\right)}\right) \cdot \Phi_{i}=0, \quad i=4 .
\end{gathered}
$$

Furthermore, Eqs. (42) and (43) can be simplified as

$$
\Phi_{i}^{\prime \prime}+\lambda_{i}^{2} \Phi_{i}=0
$$

where

$$
\begin{aligned}
& \lambda_{i}^{2}=\frac{\rho_{i} \Omega^{2}}{G_{i}}, \quad i=1,2,3,5,6, \\
& \lambda_{i}^{2}=\frac{\rho_{i} \Omega^{2}}{c_{55}^{E}+\left(e_{15}^{2} / \varepsilon_{11}^{S}\right)}, \quad i=4 .
\end{aligned}
$$

For Eq. (44), the solution reads

$$
\Phi_{i}(z)=C_{1 i} \sin \lambda_{i} z_{i}+C_{2 i} \cos \lambda_{i} z_{i}, \quad i=1, \ldots, 6,
$$

where $C_{1 i}$ and $C_{2 i}$ are the constants which are determined by satisfying the boundary and transition conditions at the ends and between the different regions of the stator. 
As the longitudinal and torsional PZT are short-circuited and both ends of the stator are free in the case of the torsional vibration, the boundary and transition conditions for this problem may be written as

$$
\begin{aligned}
& \Phi_{1}^{\prime}(0)=0, \quad \Phi_{6}^{\prime}\left(l_{6}\right)=0, \quad \Phi_{i}\left(l_{i}\right)=\Phi_{i+1}(0), \quad i=1, \ldots, 5, \\
& G_{3} \Phi_{3}^{\prime}\left(l_{3}\right)-\left(c_{55}^{E}+\frac{e_{15}^{2}}{\varepsilon_{11}^{S}}\right) \Phi_{4}^{\prime}(0)+\frac{e_{15}^{2}}{l_{4} \varepsilon_{11}^{S}}\left[\Phi_{4}\left(l_{4}\right)-\Phi_{4}(0)\right]=0, \\
& G_{5} \Phi_{5}^{\prime}(0)-\left(c_{55}^{E}+\frac{e_{15}^{2}}{\varepsilon_{11}^{S}}\right) \Phi_{4}^{\prime}\left(l_{4}\right)+\frac{e_{15}^{2}}{l_{4} \varepsilon_{11}^{S}}\left[\Phi_{4}\left(l_{4}\right)-\Phi_{4}(0)\right]=0, \\
& G_{1} \Phi_{1}^{\prime}\left(l_{1}\right)-G_{2} \Phi_{2}^{\prime}(0)=0, \quad G_{2} \Phi_{2}^{\prime}\left(l_{2}\right)-G_{3} \Phi_{3}^{\prime}(0)=0, \\
& G_{5} I_{p} \Phi_{5}^{\prime}\left(l_{5}\right)-G_{6} I_{p} \Phi_{6}^{\prime}(0)=J \Omega^{2} \Phi_{6}(0),
\end{aligned}
$$

where

$$
\begin{aligned}
& I_{p}=\frac{\pi}{32}\left(d_{2}^{4}-d_{1}^{4}\right), \\
& J=\frac{\pi a \rho}{32}\left(d_{3}^{4}-d_{2}^{4}\right) .
\end{aligned}
$$

Substitution of Eq. (47) in Eq. (48) reads

$$
\mathbf{B C}=\mathbf{r},
$$

where $\mathbf{C}=\left(C_{11}, C_{12}, \ldots, C_{16}, C_{21}, \ldots, C_{26}\right)^{\mathrm{T}}$ and $\mathbf{B}$ is the coefficient matrix. Like before, the condition

$$
\operatorname{det}(\mathbf{B})=0
$$

allows to compute the eigenfrequencies for torsional vibration.

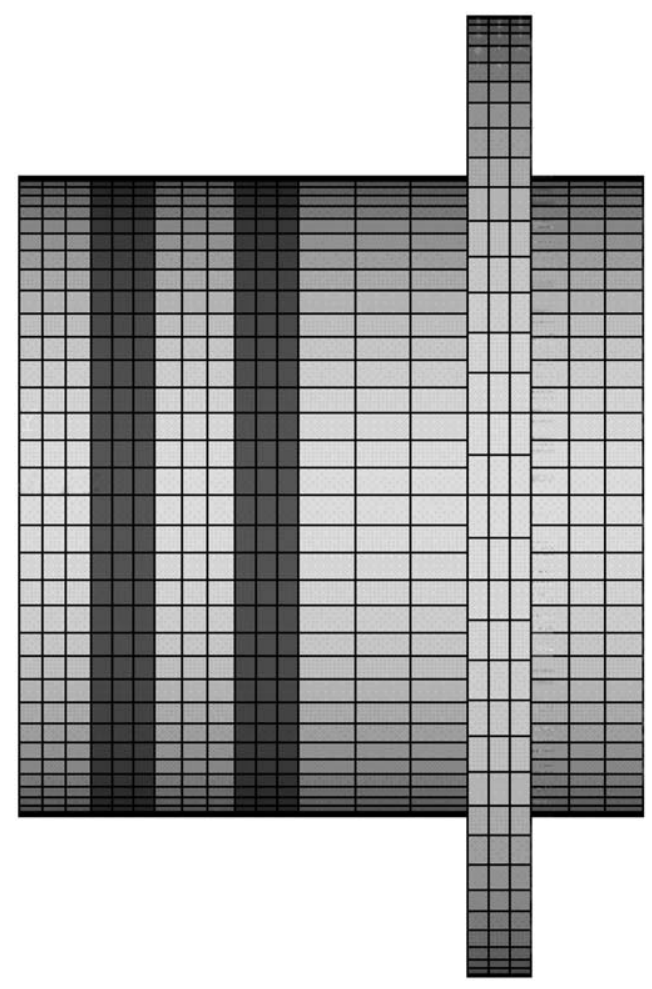

Fig. 5. FE model for vibration analysis. 


\section{Results and discussions}

The properties of the motor such as the eigenfrequencies of longitudinal and torsional vibrations may be analyzed via the analytical model. To obtain the optimized geometry of the stator and to analyze the vibration mode in more detail also an FE model is proposed (see Fig. 5). For the solution of the FE model the commercial software ANSYS is used. The material parameters for both models are given in Table 1.

A prototype of the present motor requests that the mode for the longitudinal vibration is the first mode and that for the torsional vibration is also the first mode. The symmetrical structure of the motor is supposed to produce the maximum torque if the stators operate in the range between two resonance frequencies of longitudinal and torsional vibration.

Table 1

Material parameters of the motor used in the models

\begin{tabular}{lll}
\hline Material parameters & PIC181 & PIC255 \\
\hline$\rho\left(\mathrm{kg} / \mathrm{m}^{3}\right)$ & $7.85 \mathrm{E}+03$ & $7.76 \mathrm{E}+03$ \\
$\varepsilon_{11}^{S}$ & 740 & 870 \\
$\varepsilon_{33}^{S}(\mathrm{~N} / \mathrm{Vm})$ & 624 & 680 \\
$e_{31}$ & -4.5 & -5.6 \\
$e_{33}(\mathrm{~N} / \mathrm{Vm})$ & 14.7 & 12.8 \\
$e_{15}(\mathrm{~N} / \mathrm{Vm})$ & 11.0 & 10.3 \\
$c_{11}^{E}\left(\mathrm{~N} / \mathrm{m}^{2}\right)$ & $1.523 \mathrm{E}+11$ & $1.108 \mathrm{E}+11$ \\
$c_{33}^{E}\left(\mathrm{~N} / \mathrm{m}^{2}\right)$ & $1.314 \mathrm{E}+11$ & $1.108 \mathrm{E}+11$ \\
$c_{55}^{E}\left(\mathrm{~N} / \mathrm{m}^{2}\right)$ & $2.830 \mathrm{E}+10$ & $1.909 \mathrm{E}+10$ \\
$c_{12}^{E}\left(\mathrm{~N} / \mathrm{m}^{2}\right)$ & $8.909 \mathrm{E}+10$ & $6.326 \mathrm{E}+10$ \\
$c_{13}^{E}\left(\mathrm{~N} / \mathrm{m}^{2}\right)$ & $8.547 \mathrm{E}+10$ & $6.896 \mathrm{E}+10$ \\
$c_{44}^{E}\left(\mathrm{~N} / \mathrm{m}^{2}\right)$ & $2.830 \mathrm{E}+10$ & $1.909 \mathrm{E}+10$ \\
$c_{66}^{E}\left(\mathrm{~N} / \mathrm{m}^{2}\right)$ & $3.161 \mathrm{E}+10$ & $2.383 \mathrm{E}+10$ \\
$\mathrm{Y}$ & & \\
$v$ & & \\
\hline
\end{tabular}

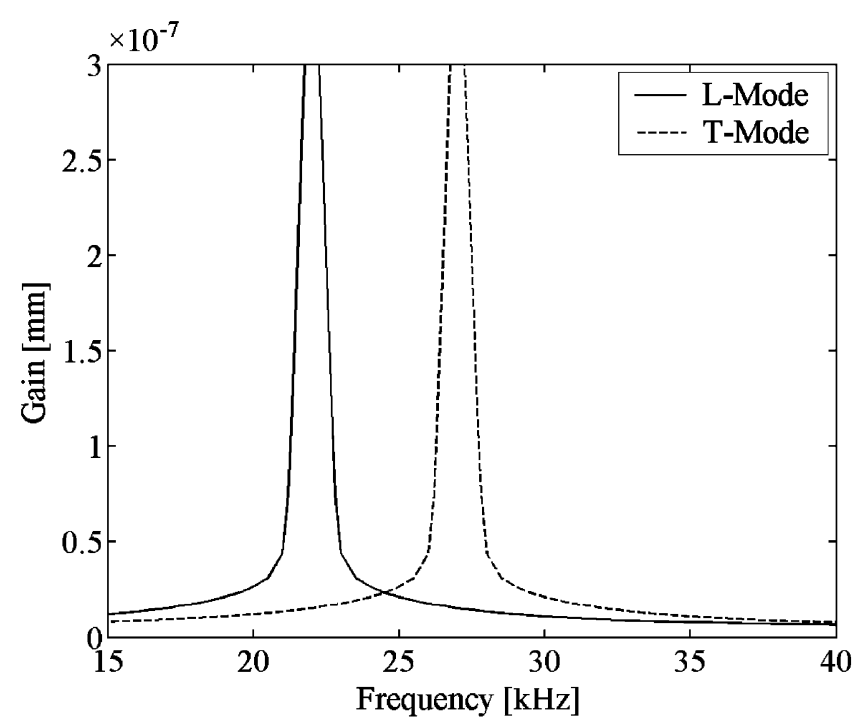

Fig. 6. Eigenfrequencies of longitudinal and torsional vibrations for the stator without ring. 
To investigate the function of the ring, the eigenfrequencies of longitudinal and torsional vibrations are calculated by the analytical model without rings at first. Thereafter, for the goal of matching the eigenfrequencies, two cases are considered: one is to change the mass of the ring and the other to change the position of the ring. The results are obtained by the analytical model and FE model.

Fig. 6 shows the first eigenfrequencies of longitudinal vibration and torsional vibration for the stator without ring. The difference of the eigenfrequencies of the stator between these two types of vibrations is about $5 \mathrm{kHz}$.

From Fig. 7 it is obvious that the first eigenfrequency of torsional vibration for the stator with a ring is decreased, while the first eigenfrequency of longitudinal vibration only changes slightly. Thus the two eigenfrequencies of the stator get closer together. The difference of these two eigenfrequencies is reduced to about $0.2 \mathrm{kHz}$. Therefore to use a ring is useful to match the eigenfrequencies of two vibration modes.

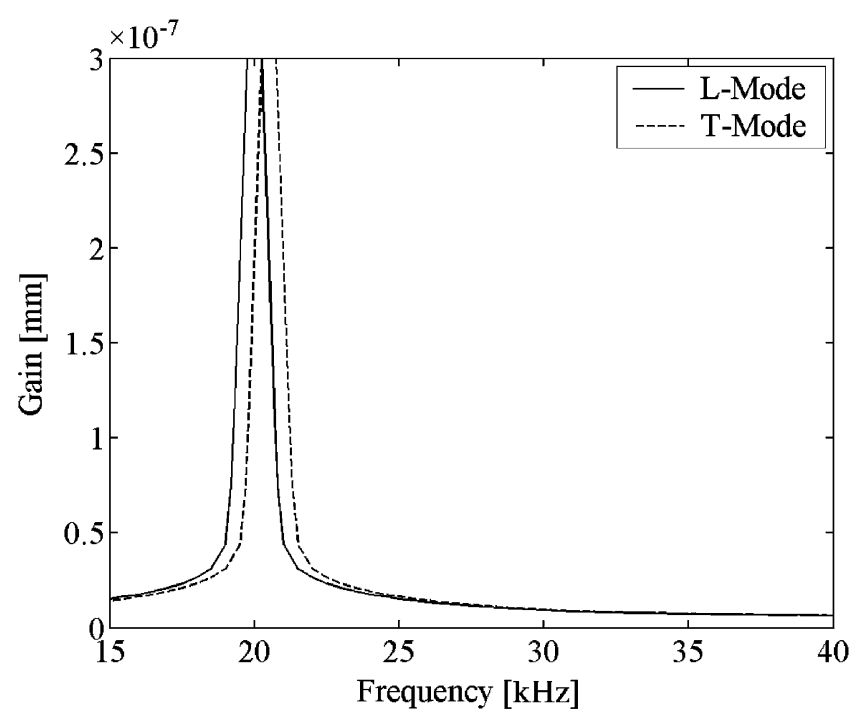

Fig. 7. Eigenfrequencies of longitudinal and torsional vibrations for the stator with a ring.

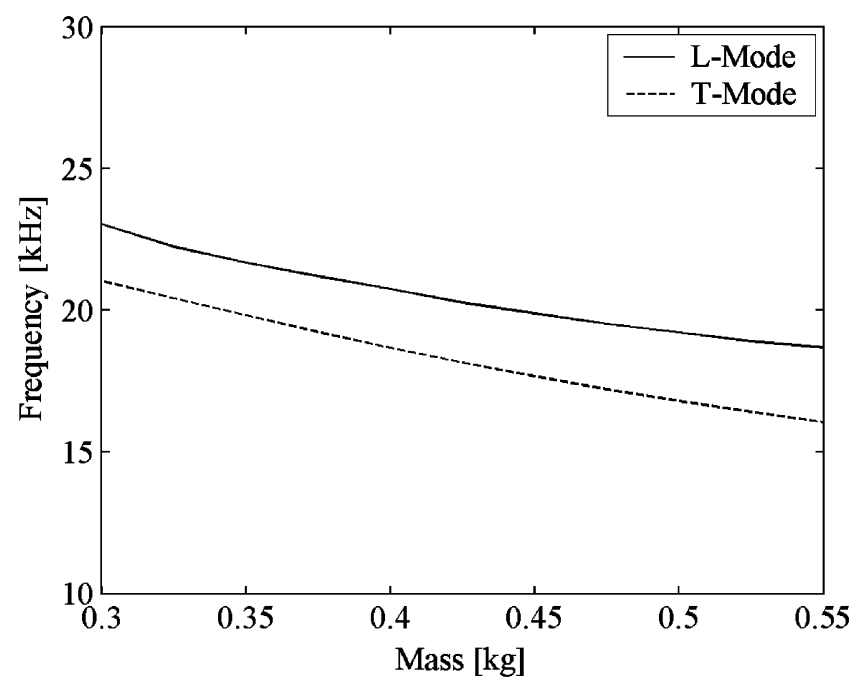

Fig. 8. Eigenfrequencies for changing mass of the ring using FE model. 


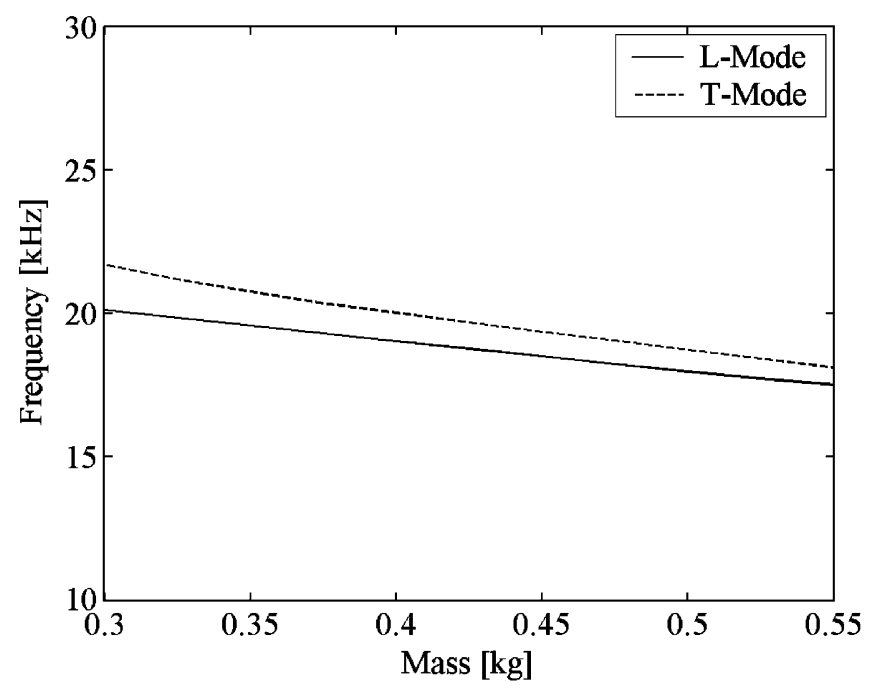

Fig. 9. Eigenfrequencies for changing mass of the ring using analytical model.

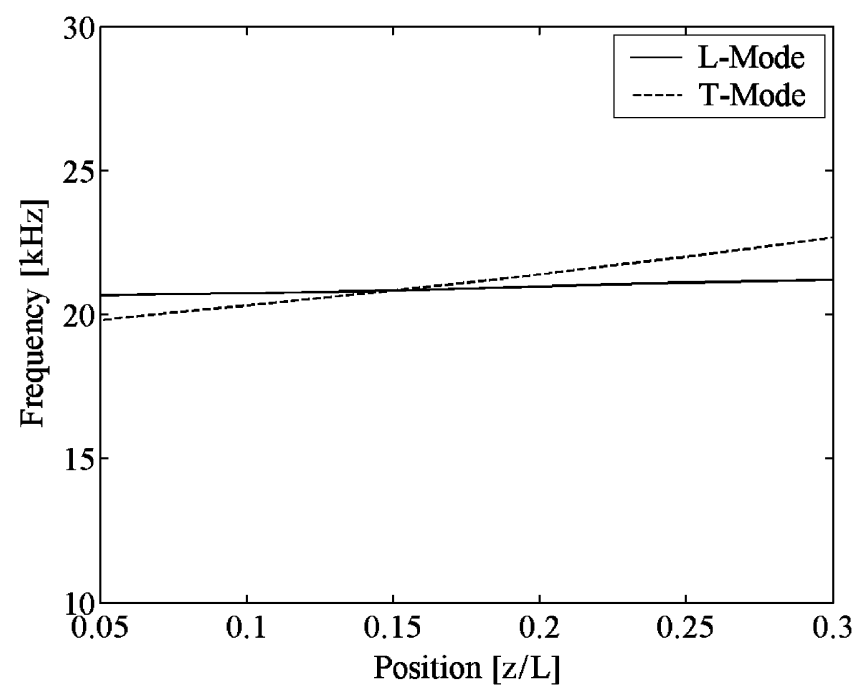

Fig. 10. Eigenfrequencies for changing position of the ring using FE model.

Figs. 8 and 9 show that the eigenfrequencies of longitudinal vibration decrease slightly with an increase of the mass of the ring. This is due to the well-known fact that the eigenfrequency is in counter proportion with the mass. The eigenfrequencies of both vibrations have the same tendency when the mass of the ring is changed. Therefore, it is not possible to obtain better effect of matching the eigenfrequencies only via changing the mass of the ring.

Figs. 10 and 11 show that when the position of the ring changes, the eigenfrequency of the longitudinal vibration only changes slightly, while the eigenfrequency of the torsional vibration has a greater change. When the ring is fixed to the anti-node of the torsional vibration, especially to the end of the stator, the decreasing value of the torsional eigenfrequency reaches the maximum. Obviously, this characteristic can be used to match the eigenfrequencies of longitudinal and torsional vibration by changing the position of the ring. Besides, the results of the theoretical model agree well with those of the FE model. 


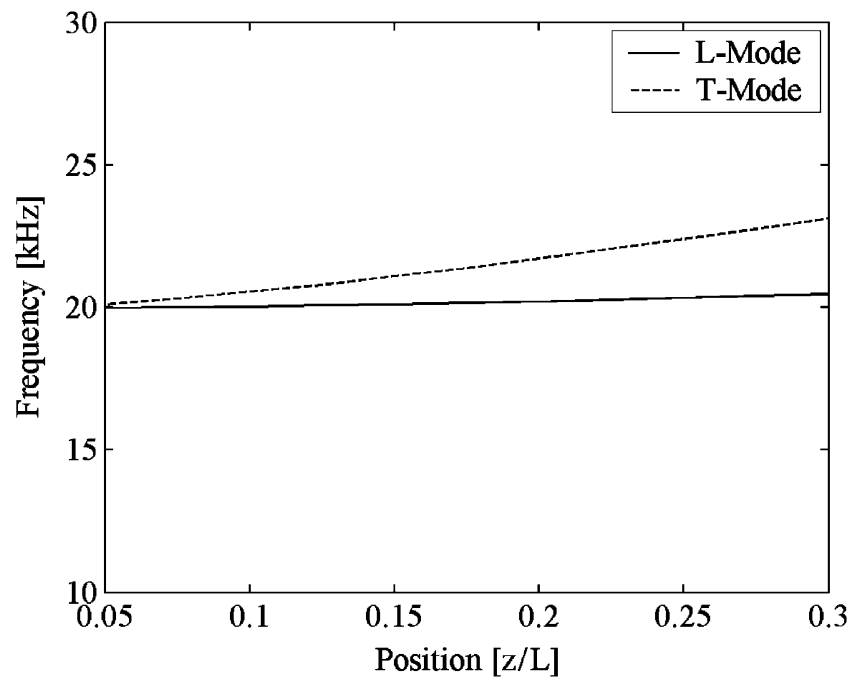

Fig. 11. Eigenfrequencies for changing position of the ring using analytical model.

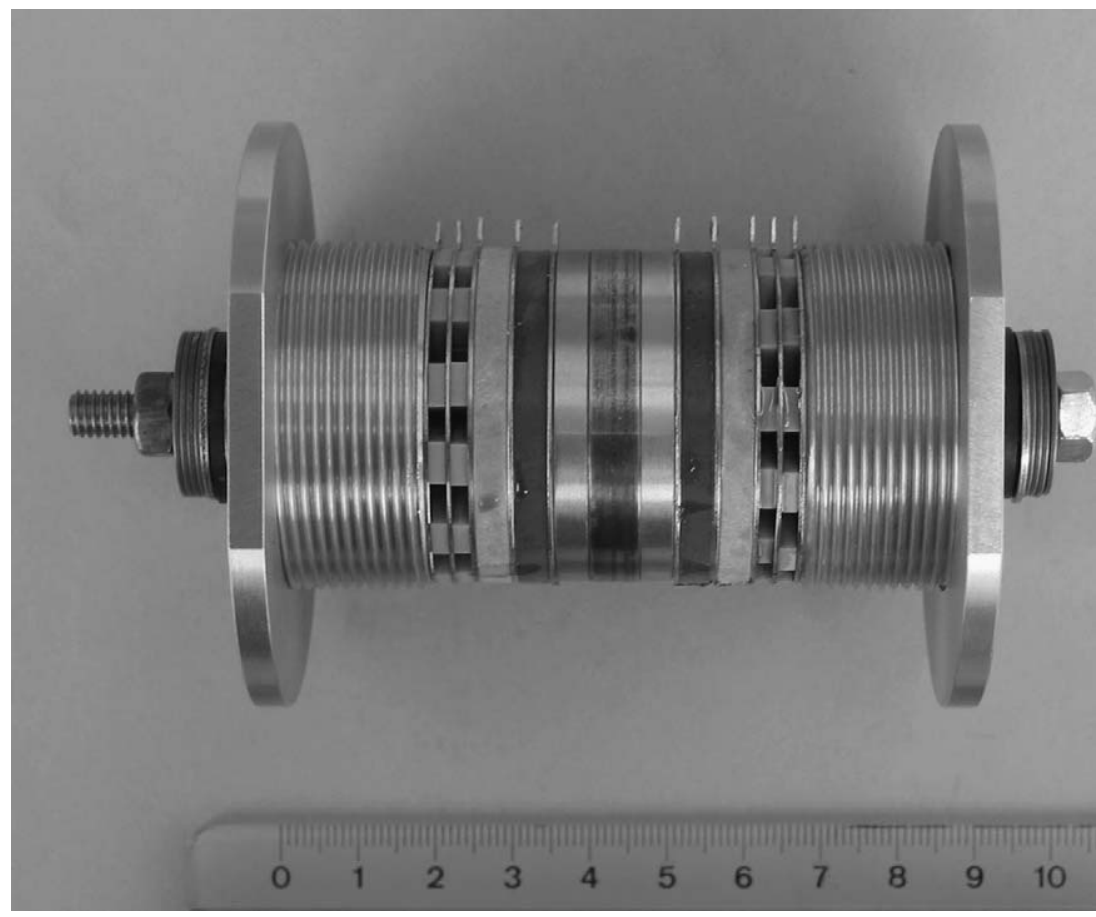

Fig. 12. The prototype of the present piezoelectric motor.

According to the analytical results, the resonant frequency of both vibrations is $20.5 \mathrm{kHz}$. The geometries of the motor $l_{1}=4.5 \mathrm{~mm}, l_{2}=4 \mathrm{~mm}, l_{3}=5 \mathrm{~mm}, l_{4}=4 \mathrm{~mm}, l_{5}=19.5 \mathrm{~mm}, l_{6}=4 \mathrm{~mm}, d_{1}=15 \mathrm{~mm}, d_{2}=40 \mathrm{~mm}$, $d_{3}=60 \mathrm{~mm}, a=4 \mathrm{~mm}$ and $b=4 \mathrm{~mm}$ are obtained by the theoretical model. The prototype of the motor is finally fabricated (see Fig. 12). 


\section{Conclusions}

The hybrid piezoelectric motor studied in this paper uses two stators, one rotor and two adjusting rings. The analysis via an analytical model and FE model show that the design of motor using this symmetrical structure is helpful to match the eigenfrequencies of longitudinal and torsional vibration. When a ring is added to the stator, the torsional eigenfrequency changes much more than that of the longitudinal vibration. The eigenfrequencies of two vibrations are not sensible to the mass of the ring, but sensible to the position of the ring.

The eigenfrequencies of longitudinal and torsional vibrations can be matched through changing the position of the adjusting ring and the working frequency of the motor can be obtained. When the motor operates at the coinciding eigenfrequency of longitudinal and torsional vibrations, while the counter-phase mode of the stators are used as well, the motor will gain the maximum torque.

\section{Acknowledgments}

This paper has benefited from the discussions with Dipl.-Ing. Andreas Maier, whose help is gratefully acknowledged.

\section{References}

[1] K. Nakamura, M. Kurosawa, S. Ueha, Characteristics of a hybrid transducer-type ultrasonic motor, IEEE Transactions on Ultrasonics, Ferroelectrics, and Frequency Control 38 (3) (1991) 188-193.

[2] M. Kurosawa, S. Ueha, Hybrid transducer type ultrasonic motor, IEEE Transactions on Ultrasonics, Ferroelectrics, and Frequency Control 38 (3) (1991) 89-92.

[3] K. Nakamura, M. Kurosawa, S. Ueha, Design of a hybrid transducer type ultrasonic motor, IEEE Transactions on Ultrasonics, Ferroelectrics, and Frequency Control 40 (4) (1993) 395-401.

[4] W. Seemann, A linear ultrasonic traveling wave motor of the ring type, Smart Materials and Structures 5 (1996) 361-368.

[5] S. Ueha, Y. Tomikawa, Ultrasonic Motors-Theory and Applications, Clarendon Press, Oxford, 1993.

[6] T.Y. Zhou, L.Q. Zhang, Y. Xue, X.Y. Chen, Resonant frequencies into degeneration of hybrid longitudinal and torsional ultrasonic motor vibration system, Piezoelectrics and Acoustooptics 21 (2) (1999) 150-160.

[7] S.Y. Lin, Study on the sandwiched piezoelectric ultrasonic torsional transducer, Ultrasonics 32 (6) (1994) $461-464$.

[8] W. Seemann, R. Gausmann, Transmission and reflection coefficients for longitudinal waves obtained by a combination of refined rod theory and FEM, Journal of Sound and Vibration 197 (5) (1996) 571-587.

[9] W. Seemann, R. Gausmann, A refined model for piezoelectric transformer, ASME 2001 Design Engineering Technical Conferences and Computers and Information in Engineering Conference, Pittsburgh, Pennsylvania, USA, September 9-12, 2001, pp. 1-10.

[10] T. Sashida, T. Kenjo, An Introduction to Ultrasonic Motors, Clarendon Press, Oxford, 1993. 